\title{
A VIRTUAL MICROFLUIDIC CHANNEL WITH OIL-REPELLANT SURFACES FOR GUIDING LOW SURFACE TENSION LIQUIDS
}

\author{
Riberet Almeida and Jae Wan Kwon
}

University of Missouri-Columbia, USA

\begin{abstract}
This paper reports for the first time a novel technique to form a 'virtual' microfluidic channel for guiding low surface tension liquids. Confining and manipulating non-aqueous low surface tension liquids within a desired area are not trivial but pose great challenges in microfluidic devices. Newly developed oil-repellant surfaces formed by using a plasma assisted surface micromachining process have been employed to control the surface wettability for low surface tension liquids. Such oleophophobic surfaces have been utilized to form microfluidic channels without sidewalls. Various low surface tension liquids have been electrically actuated and successfully guided along the 'virtual' microfluidic channel.
\end{abstract}

\section{INTRODUCTION}

Control of surface wettability for manipulating liquids has been widely adapted in many practical applications [1]. Although manipulating and controlling aqueous solutions are relatively easy, it is very difficult to prevent spreading of non-aqueous low surface tension liquids (oils including decane, octane, and hexadecane) onto non-desired regions. In addition, superoleophobic surfaces inside microfluidic channels have not been broadly explored yet. Moreover, inherently oleophobic $\left(\theta>90^{\circ}\right.$ on a smooth surface) surface chemistries are naturally unavailable. This means that the Wenzel state lowers the contact angle further, while the Cassie state is inherently unstable on most geometries [2]. Unlike superhydrophobic surfaces, superoleophobic surfaces cannot be easily fabricated with nano-structures [3]. And the use of micro/nano-structured features to prevent lateral spreading of low surface tension liquids has not been very effective. The relationship between oleophilic surfaces and superoleophobic surfaces is more complex than the relationship between hydrophilic and superhydrophobic surfaces [2]. In fact, the surface chemistry of the previously reported superoleophobic surfaces using various fluorinated coatings over re-entrant geometries is actually slightly oleophilic [2]. When the oil drop falls into the Wenzel state due to perturbation, the oil drop can simply be pinned or spread laterally. Thus, it is not clear how oleophobically stable the surface is to lateral flows. We have developed an oil-repellent surface which can resolve the concerns described above.

\section{OIL REPELLANT SURFACE}

Since the oleophobicity is governed by the surface geometry, we designed an oil-repellant surface by combining laterally and vertically oriented re-entrant geometries to liquids.

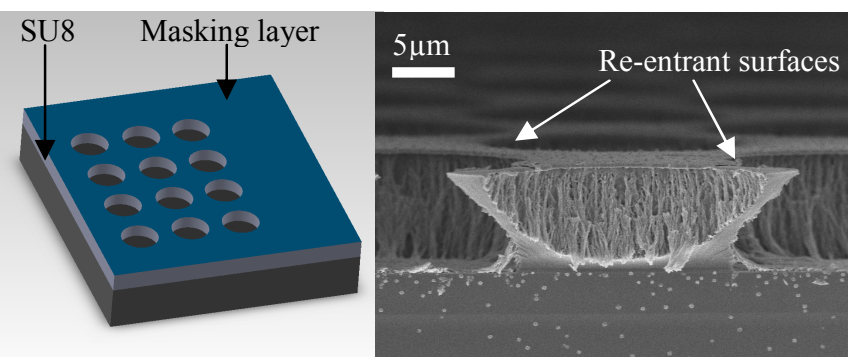

Figure 1: Schematic view and SEM image of micromachined features comprising the oil-repellant surface.
Thus, such surfaces can prohibit laterally approaching low surface tension liquids. In order to minimize $\phi_{\mathrm{s}}$, which is the areal fraction of the liquid-air interface, occluded by the texture in the CassieBaxter model, we utilized surface micromachined features as shown in Fig. 1. The geometry of the features can act as oilrepelling features to the lateral flow of low surface tension liquids. The re-entrant features in the textured area formed by reactive ion etching can clearly be seen in the SEM image. By application of the very uniquely micromachined features with a hydrophobic coating, selective wetting by a non-aqueous low surface tension liquid has been successfully achieved as illustrated in Fig. 2 .
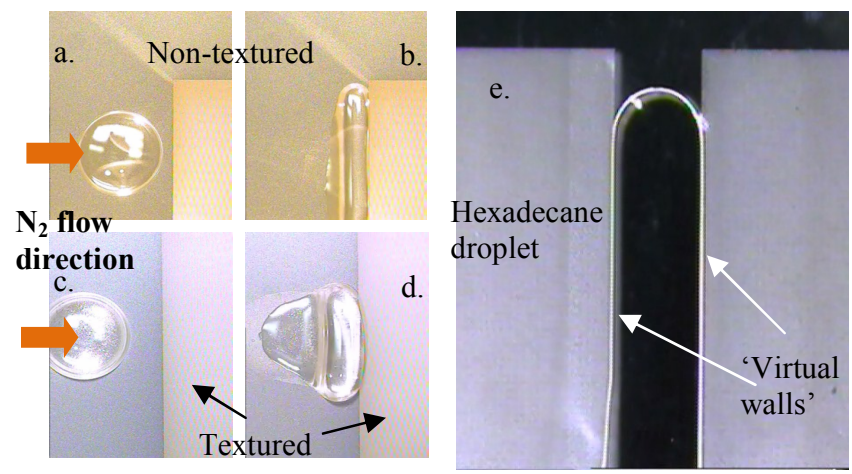

Figure 2: Silicone oil droplet (a, b) and castor oil droplet $(c, d)$ pneumatically actuated $(a \rightarrow b, c \rightarrow d)$ from non-textured towards the textured oil-repellant region. e. Hexadecane moves along an un-textured path due to the presence of 'virtual' walls.

As can be seen in the above image, a drop of hexadecane selectively wets the non-textured region. The textured regions turn out to be too oil-repellant to allow the hexadecane droplet to wet the surface. Even when pushed by a strong pneumatic force, the oil droplet cannot move over the textured region. Various low surface tension liquids introduced between the textured areas have been well confined within the oleophilic pathways as shown in Fig. 2e. The gas-liquid interface is pinned precisely at the boundary between the textured and non-textured regions. Because there are no physical walls on the sides of the liquid stream, it can be looked upon as confinement by the presence of 'virtual' walls.

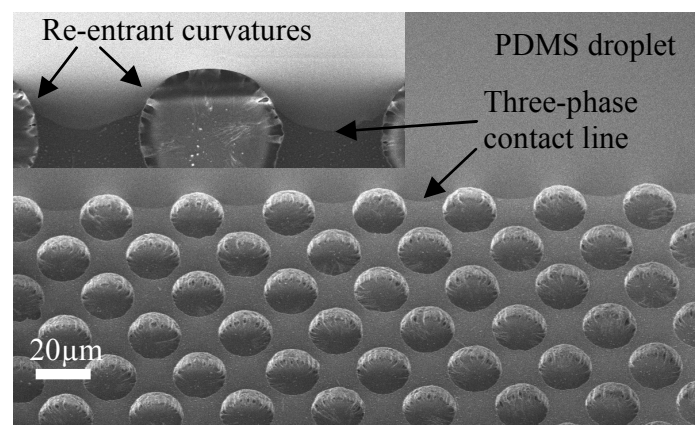

Figure 3: PDMS droplet at the edge of an oil-repellant surface for mimicking the behavior of the oil droplet three-phase contact line at these features.
Solid-State Sensors, Actuators, and Microsystems Workshop Hilton Head Island, South Carolina, June 3-7, 2012 
The oil-repellant behavior arises most importantly from the interaction of the three-phase contact line with the re-entrant geometries of the micromachined features on the surface. A further study about the interaction of the three-phase contact line with the boundary between the textured and non-textured areas was carried out by using scanning electron microscopy (SEM). For this, a PDMS droplet, which has a similar surface tension to that of silicone oil, was placed on the non textured surface and pneumatically pushed towards the micro textured area and then cured. Clear images of the representation of an oil droplet by PDMS at the boundary were obtained as shown in Fig. 3. The fluidic resistance faced by the three-phase contact line at the boundary prevents its further advancement.

\section{VIRTUAL MICROFLUIDIC CHANNEL WITH BUILT-IN LIQUID DRIVERS}

This phenomenon has been further exploited to form a 'virtual' microfluidic channel for guiding low surface tension liquids. In order to drive the low surface tension liquid confined between two 'virtual' walls, we have implemented electrodes only under the non-textured regions to provide the necessary dielectrophoretic forces. The brief fabrication steps are shown in Fig. 4.

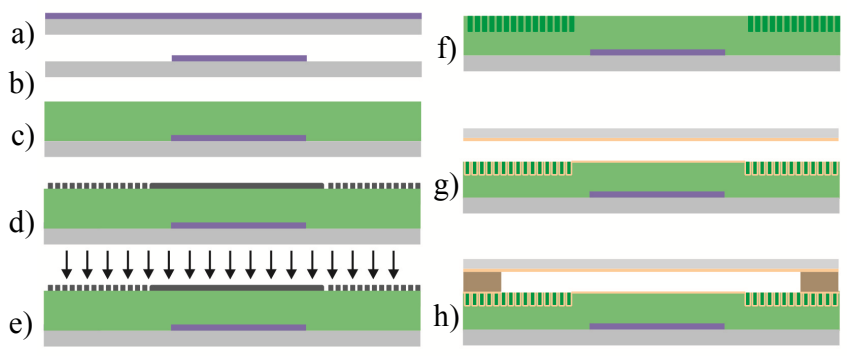

Figure 4: Fabrication steps outlined a) Metallization b) Patterning of metal layer to define electrodes c) SU8 layer d) Metallization and patterning defining textures e) $\mathrm{O}_{2}$ RIE etch f) Stripping of metal layer g) Coating substrate surface and coverslip with hydrophobic layer h) Final device assembly via spacers.

Fabrication starts with the patterning of a metalized glass substrate which defines the underlying dielectrophoretic (DEP) electrodes. On top of these electrodes, a $10 \mu \mathrm{m}$ thick SU8 layer was deposited and cured followed by a metallization and patterning step. The patterned metalized layer acts as a mask to the subsequent surface micromachining step which defines the oilrepellant features. Surface micromachining of the cured SU8 was carried out by etching in a reactive ion etcher (RIE) with oxygen. The micromachined surface as well as the top cover glass substrate was then made hydrophobic with the application of a very thin Cytop layer (100nm). The top cover substrate was then assembled over a $25 \mu \mathrm{m}$ thick spacer and bonded. Since the electrodes are not in direct contact with the liquids, we believe that dielectric as well as conducting liquids can be driven along the virtual microfluidic channel. As soon as a test liquid was loaded into the device, the liquid remained confined between the 'virtual' walls. Compared to viscous fluids such as silicone and castor oil, less viscous fluids such as hexadecane and Fluorinert FC-70 flow more easily along the paths confined by the textured oil-repellant surfaces. Activation of the electrodes with a DC voltage source, thereby generating dielectrophoretic forces in the oil droplet cause it to move along the 'virtual' microfluidic channel (non-textured area) as shown in
Fig. 5. The liquid-air interfaces along the 'virtual' walls still look clearly straight. In addition, the actuation voltages for various test liquids with different properties are summarized in Table 1.

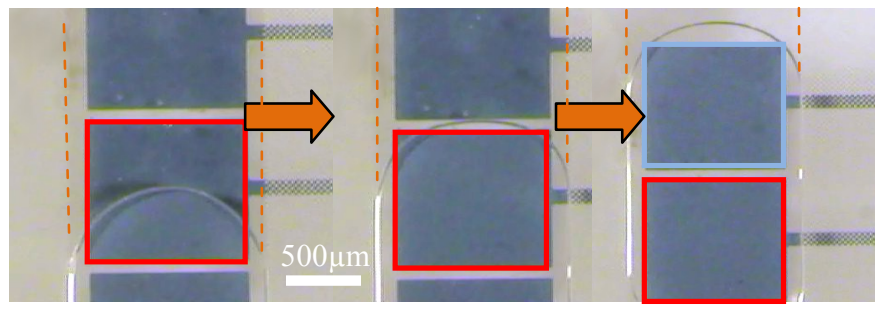

Figure 5: Droplet (Silicone oil) actuated across the desired path using liquid Dielectrophoresis (L-DEP) by actuating one electrode at a time.

\section{CONCLUSION}

A virtual microfluidic channel for low surface tension liquids has been successfully demonstrated. An oil-repellant surface was first designed and tested. Implementation of 'virtual' walls generated by the oil-repellant surfaces was carried out to confine low surface tension liquids. Electrical actuation of various test liquids between the 'virtual' walls has shown successful outcomes. This technique can be applied to many microfluidic devices dealing with various low surface tension liquids.

Table 1: Actuation voltages with and without the use of micro textured guiding of various low surface tension liquids

\begin{tabular}{|l|c|c|c|c|}
\hline Test liquids & $\begin{array}{c}\text { Actuation } \\
\text { voltage } \\
\text { without } \\
\text { micro } \\
\text { textures }\end{array}$ & $\begin{array}{c}\text { Actuation } \\
\text { voltage } \\
\text { with } \\
\text { micro } \\
\text { textures }\end{array}$ & $\begin{array}{c}\text { Di- } \\
\text { electric } \\
\text { constant }\end{array}$ & $\begin{array}{c}\text { Surface } \\
\text { tension } \\
(\mathbf{m J} / \\
\left.\text { cm }^{2}\right)\end{array}$ \\
\hline $\begin{array}{l}\text { Silicone oil } \\
(50 c S t)\end{array}$ & $310 \mathrm{~V}$ & $325 \mathrm{~V}$ & 2.75 & 20.3 \\
\hline $\begin{array}{l}\text { Castor oil } \\
(985 \mathrm{cSt})\end{array}$ & $316 \mathrm{~V}$ & $450 \mathrm{~V}$ & 4.7 & 39 \\
\hline $\begin{array}{l}\text { Hexa- } \\
\text { decane } \\
(3.02 \mathrm{cSt})\end{array}$ & $\sim 400 \mathrm{~V}$ & $420 \mathrm{~V}$ & 2.05 & 27.4 \\
\hline $\begin{array}{l}\text { FC-70 } \\
(24 \mathrm{cSt})\end{array}$ & $188 \mathrm{~V}$ & $210 \mathrm{~V}$ & 1.98 & 18 \\
\hline
\end{tabular}

\section{ACKNOWLEDGEMENT}

This research was supported by the National Science Foundation under Grant No. ECCS 0901618.

\section{REFERENCES}

[1] N. Verplanck, et al., "Wettability switching techniques on superhydrophobic surfaces," Nanoscale Research Letters, vol. 2, pp. 577-596, 2007.

[2] V. Jokinen, et al., "Controlled Lateral Spreading and Pinning of Oil Droplets Based on Topography and Chemical Patterning," Langmuir, 2011.

[3] A. Tuteja, et al., "Robust omniphobic surfaces, "Proceedings of the National Academy of Sciences, vol. 105, p. 18200 , 2008 .

CONTACT R. Almeida;+1-573-884-7867; rab5b@mail.missouri.edu 